\title{
Editorial
}

\section{Fault Diagnosis and Prognosis of Critical Components}

\author{
Dong Wang, ${ }^{1}$ Chuan Li, ${ }^{2}$ Achmad Widodo, ${ }^{3}$ \\ Pavan Kumar Kankar, ${ }^{4}$ and Wahyu Caesarendra ${ }^{5}$ \\ ${ }^{1}$ City University of Hong Kong, Kowloon Tong, Hong Kong \\ ${ }^{2}$ Universidad Politécnica Salesiana, 010150 Cuenca, Ecuador \\ ${ }^{3}$ University of Diponegoro, Semarang 50275, Indonesia \\ ${ }^{4}$ PDPM Indian Institute of Information Technology, Jabalpur 482005, India \\ ${ }^{5}$ University of Wollongong, Wollongong, NSW 2522, Australia \\ Correspondence should be addressed to Dong Wang; dongwang4-c@my.cityu.edu.hk
}

Received 12 November 2015; Accepted 12 November 2015

Copyright ( 92016 Dong Wang et al. This is an open access article distributed under the Creative Commons Attribution License, which permits unrestricted use, distribution, and reproduction in any medium, provided the original work is properly cited.

Some critical components, such as bearings, gearboxes, and impellers, are widely used in machines. Their faults may accelerate failures of other components and finally result in machine breakdowns. To prevent any unexpected machine breakdowns and accidents, early faults of critical components should be detected as soon as possible. Once early faults of critical components are diagnosed, their performance degradation assessment and remaining useful life estimation should be conducted to maximize lifetime of critical components. This special issue mainly focuses on fault diagnosis and prognosis of critical components and enables researchers and engineers to share their most recent developments and ideas so as to improve technologies adopted in the research community of machine fault diagnosis and prognosis.

We have received many submissions from different countries and regions. After rigorous peer review processes, the following manuscripts have been accepted and their contents are briefly summarized as follows.

In the paper entitled "Gearbox Fault Identification and classification with Convolutional Neural Networks," Z. Chen et al. proposed a deep learning convolutional neural network based intelligent method for gearbox fault diagnosis. The comparison with the classic support vector machine based diagnostic method demonstrated that their method has higher fault prediction accuracies.

In the paper entitled "Rolling Element Bearing Fault Diagnosis Based on Multiscale General Fractal Features," W. Wen et al. proposed a multiscale general fractal dimensions based method to identify different bearing faults under different operating conditions and they experimentally demonstrated that their method has better performance than wavelet packet and empirical mode decomposition based methods under different operating conditions.

In the paper entitled "Condition Monitoring and Fault Diagnosis for an Antifalling Safety Device," G. Yang and H. Liang proposed a discrete wavelet transform based noise reduction method for condition monitoring and fault diagnosis of an antifalling safety device used in a construction elevator. Moreover, they experimentally found that the sym 8 wavelet basis is the most effective wavelet basis among some wavelet basis candidates.

In the paper entitled "Screw Performance Degradation Assessment Based on Quantum Genetic Algorithm and Dynamic Fuzzy Neural Network," X. Zhang et al. used quantum genetic algorithm to optimize dynamic fuzzy neural network so as to propose a screw performance degradation assessment method. The results show that the proposed method has higher prediction accuracies than backpropagation neural network and radial basis function neural network.

In the paper entitled "A New Transmissibility Based Indicator of Local Variation in Structure and Its Application for Damage Detection," X. Z. Li et al. proposed a new transmissibility based indicator to monitor local variations of stiffness and damping for structure health condition monitoring. The results demonstrated that their proposed indicator is effective in localizing cracks of beam structures.

In the paper entitled "Planetary Gearbox Vibration Signal Characteristics Analysis and Fault Diagnosis," Q. Miao and 
Q. Zhou proposed a planetary vibration model to understand planetary vibration components. The results showed that their proposed model matches vibration data collected from a planetary test rig.

In the paper entitled "Multifault Diagnosis of Rolling Element Bearings Using a Wavelet Kurtogram and Vector Median-Based Feature Analysis," P. H. Nguyen and J.-M. Kim proposed a wavelet kurtogram and vector medianbased feature analysis method to diagnose different bearing faults under different rotational speeds and crack sizes. Compared with principle component analysis, independent component analysis, and linear discriminant analysis, their proposed analysis method has better performance for feature extraction.

In the paper entitled "Fault Diagnosis for a Multistage Planetary Gear Set Using Model-Based Simulation and Experimental Investigation," G. Li et al. proposed a coupled lateral and torsional dynamic model to predict modulation sidebands of a two-stage compound planetary gear set. Moreover, fault characteristic frequencies were derived. Their work provided a theoretical support for fault diagnosis of a multistate planetary gear set by using modulation sideband analysis.

In the paper entitled "In Situ Measurement of Seeking Speed and Seeking Induced Head-Disk Interface Instability in Hard Disk Drives," Y. Wang et al. investigated the instability of head-disk interface caused by the voice coil motor end crashing the crash stop in the process of magnetic head seeking and they proposed a maximum likelihood estimation and extended Kalman filter based method to seek speed. The results show that the rolling mode of air bearing will lead to a serious disk damage in the case of high seeking speed.

In the paper entitled "Cyclostationary Analysis for Gearbox and Bearing Fault Diagnosis," Z. Feng and F. Chu derived explicit expressions of cyclic correlation and cyclic spectrum of modulation and frequency modulation signals and thoroughly investigated their applications to gearbox and bearing fault diagnosis.

In the paper entitled "Sparse Signal Representations of Bearing Fault Signals for Exhibiting Bearing Fault Features," W. Peng et al. proposed an optimal wavelet filtering based sparse signal presentation method. The results demonstrated that their proposed method is effective in using a few wavelet coefficients to represent bearing fault features.

In the paper entitled "Feature Extraction of Faulty Rolling Element Bearing under Variable Rotational Speed and Gear Interferences Conditions," D. Zhao et al. proposed an instantaneous dominant meshing multiply and empirical mode decomposition based on bearing fault feature extraction method under varying operation conditions. Multiple vibration mixtures were used to verify the effectiveness of their proposed method.

In the paper entitled "Two General Architectures for Intelligent Machine Performance Degradation Assessment," Y. Xu et al. proposed two general architectures for intelligent machine performance degradation assessment. Their proposed architectures have wide applications, such as gearbox performance degradation and bearing performance degradation.
In the paper entitled "Application of Reassigned Wavelet Scalogram in Wind Turbine Planetary Gearbox Fault Diagnosis under Nonstationary Conditions," X. Chen and Z. Feng applied reassigned wavelet scalogram to diagnose wind turbine planetary gearboxes. The experimental and in situ evaluations were conducted to verify the effectiveness of reassigned wavelet scalogram for turbine planetary gearbox fault diagnosis.

In the paper entitled "Research on the Sparse Representation for Gearbox Compound Fault Features Using Wavelet Bases," C. Luo et al. proposed a split augmented Lagrangian shrinkage algorithm based method to extract gearbox compound fault features. Laplace wavelet optimized by correlation filtering was used to design a dictionary for constructing redundant basis functions. The results demonstrated the effectiveness of their proposed method and showed that a series of sparse coefficients are able to show gearbox compound fault features.

In the paper entitled "A Hybrid Prognostic Approach for Remaining Useful Life Prediction of Lithium-Ion Batteries," W.-A. Yang et al. proposed a selective kernel ensemblebased relevance vector machine and particle swarm optimization based prognostic method for remaining useful life prediction. Their proposed method has potential applications to prognostics of gearbox, bearing, and so on.

In the paper entitled "Application of Artificial Neural Network for Damage Detection in Planetary Gearbox of Wind Turbine," M. Straczkiewicz, and T. Barszcz proposed an artificial neural network and linear regression based method to distinguish healthy and damaged planetary gearbox conditions. Their proposed method has great potential for condition monitoring of various rotating machinery, such as mining excavators and gas turbines.

In the paper entitled “Trace Ratio Criterion-Based Kernel Discriminant Analysis for Fault Diagnosis of Rolling Element Bearings Using Binary Immune Genetic Algorithm," W.-A. Yang et al. proposed a trace ratio criterion-based kernel discriminant analysis based method for bearing fault diagnosis. The parameters of their proposed method were optimized by binary immune genetic algorithm. The results showed that their proposed method is better than some popular intelligent methods for bearing fault diagnosis.

In the paper entitled "Gearbox Fault Diagnosis Using Complementary Ensemble Empirical Mode Decomposition and Permutation Entropy," L. Zhao et al. proposed a complementary ensemble empirical mode decomposition and permutation entropy based method for gearbox fault diagnosis. The results showed that their proposed method is able to improve prediction accuracies of support vector machines.

We sincerely hope that this special issue could provide useful technologies and information for researchers and engineers in the research community of fault diagnosis and prognosis. 


\section{Acknowledgments}

We would like to thank all authors for submitting their works to this special issue. We also would like to thank all reviewers and editors for their valuable comments on all manuscripts.

Dong Wang Chuan $\mathrm{Li}$ Achmad Widodo

Pavan Kumar Kankar Wahyu Caesarendra 


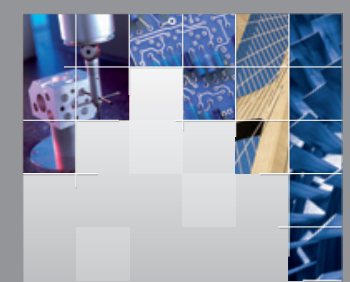

\section{Enfincering}
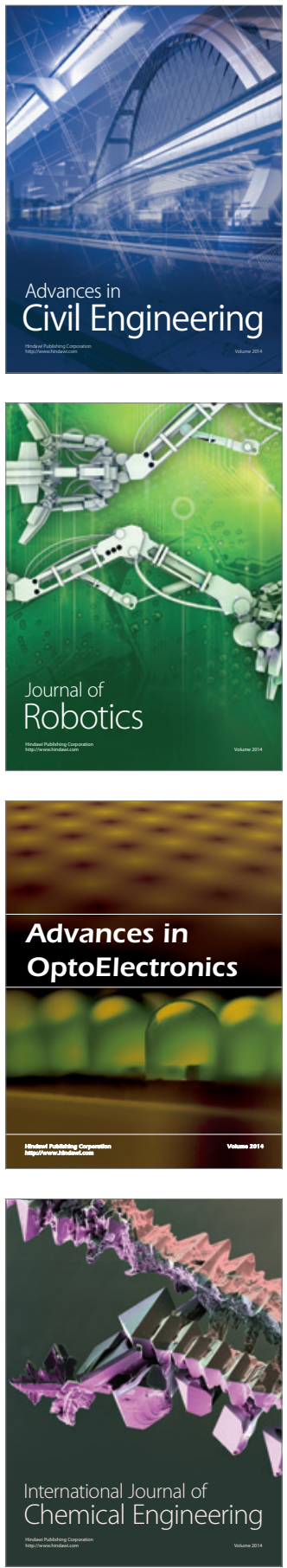

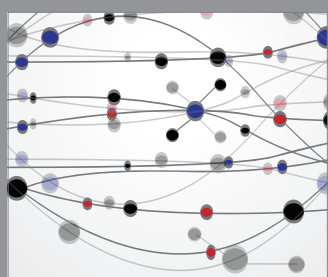

The Scientific World Journal

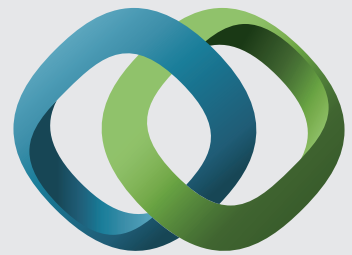

\section{Hindawi}

Submit your manuscripts at

http://www.hindawi.com
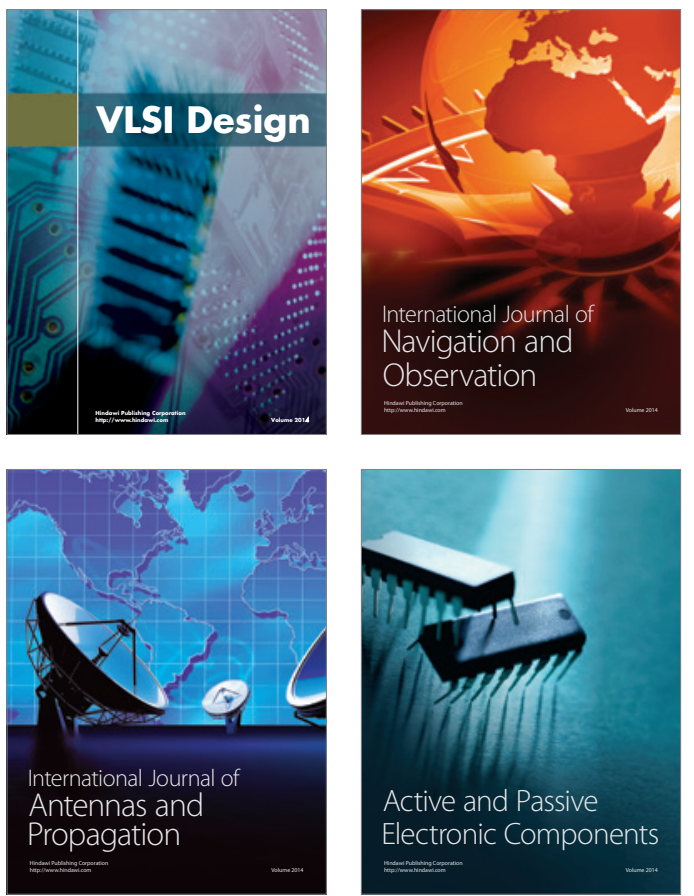
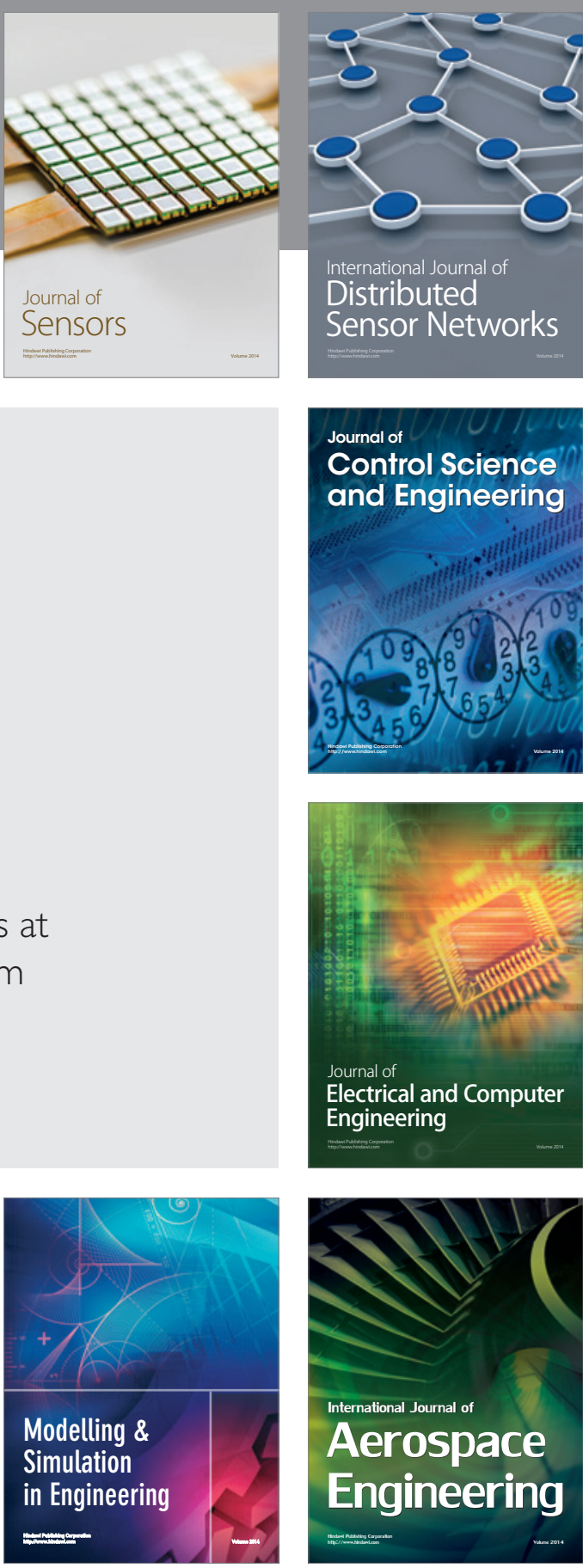

International Journal of

Distributed

Sensor Networks

Journal of

Control Science

and Engineering
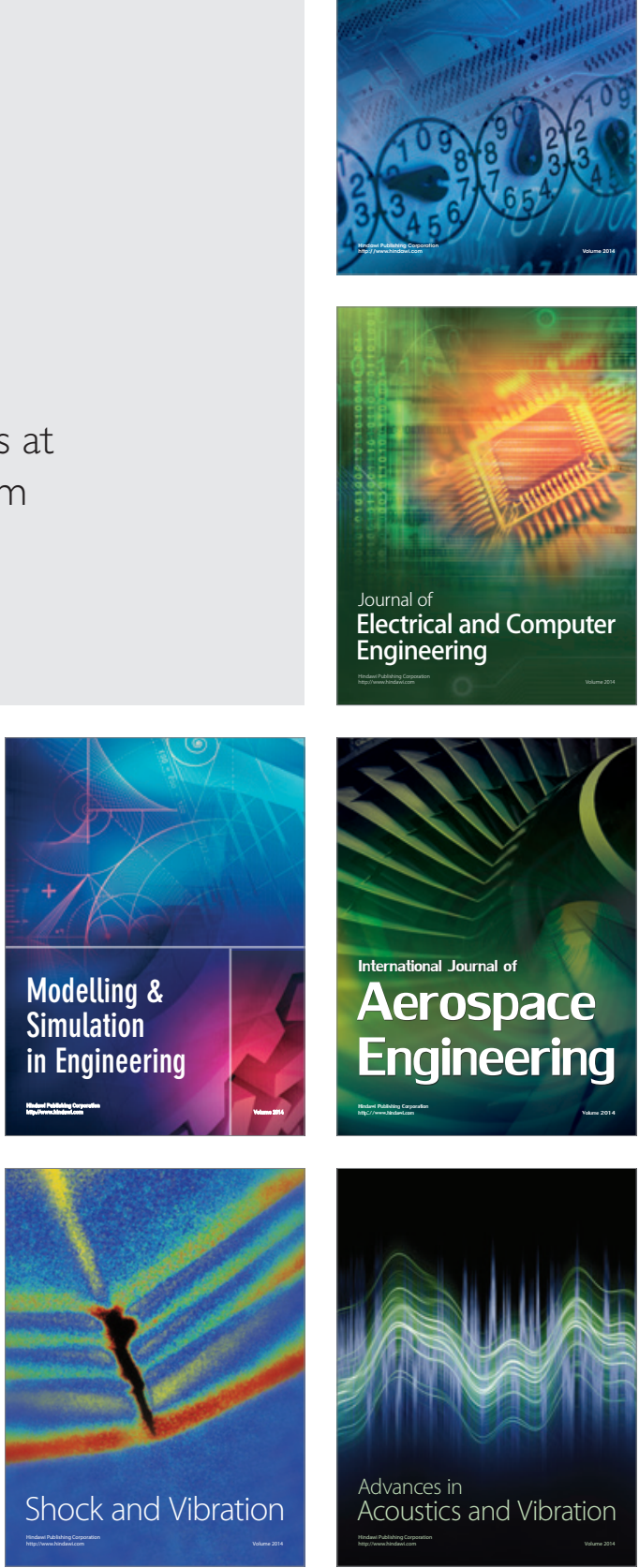